\title{
A Comparison of Subject and Institutional Repositories in Self-archiving Practices
}

\author{
Jingfeng Xia
}

\section{Introduction}

In regard to the mechanism of content recruitment, institutional repositories (IR) at their initial stage of development in the early 2000s followed what subject-based repositories (SR) had already practiced for many years. Since then, self-archiving has become the primary way of aggregating digital collections for IRs, although recent operations and content materials of IRs have been more diverse. Inheriting the nature of self-archiving from SRs, IRs are thought to see the same practices by faculty authors. It is further assumed that, because digital repositories are available and successful in only a few scientific disciplines, scholars in the disciplines where a SR has been in existence tend to be more active in contributing to an IR than scholars of other disciplines who are unfamiliar with self-archiving previously. Such a correlation between disciplinary culture and the willingness of faculty to self-archive in IRs has been theorized extensively in the literature of digital repositories.

In general, the theory has practical importance for the development and management of IRs. For a long time, repository managers and administrators have been bothered by the reluctance of faculty to participate in the archiving of their research output into IRs and therefore by the small content size of their IR databases. Various strategies have been developed to solve the problem, which are mainly based on the understanding of information input and use behavior, particularly of faculty who are the major target of institutional repositories.

In recent months, a much discussed topic has been the adoption of a mandate policy in repository management to require scholars to archive their research. In the United States, the Congress passed a law demanding self-archiving of research results supported by the NIH grants into PubMed - a famous SR in biomedical science - which was signed by President Bush in late 2007. ${ }^{1}$ Several months later, faculty in arts and sciences at Harvard University voted to allow the university to make their scholarly articles available free online through its institutional repository, which was joined lately by the Harvard Law School. ${ }^{2}$ These are big, positive steps for open access movement with dramatic impact on the development of digital repositories. However, it still remains questionable about how such mandate policies can be implemented in practice. Outside the U.S., the mandate policies of self-archiving have been in place for some years such as in Australia and Britain. ${ }^{3}$ New policies have become increasingly popular for promoting open access (OA), e.g., Scotland's Queen Margaret University adopted an OA mandate in February 2008, ${ }^{4}$ and the Irish Research Council for Science, Engineering and Technology announced its mandatory requirements for research papers published by its funding recipients to be made available in an OA repository, within six months of their first publication. ${ }^{5}$ EPrints.org maintains a full list of OA policies registered by individual institutions. ${ }^{6}$ It will be interesting to observe how scholars in certain fields react to such a mandatory requirement in an IR environment.

In order to test the subject culture theory, it is necessary to compare the practices of self-archiving between SRs and IRs by scholars in the same academic discipline; and it is ideal if the comparison is carried out with the assessment of an IR where a mandate policy has been implemented. This study is a 
preliminary investigation that focuses on one scientific field - physics - for the reason that it has one of the oldest and most successful SRs in the world. Physicists have long enjoyed the tradition of sharing their research ideas and results with peer researchers in the format of pre-print through an online archive database - arXiv. ${ }^{7}$ If the theory of disciplinary culture is applicable, one will expect to see that physicists have contributed as many articles to the IR with which they are affiliated as to arXiv. This study chooses physicists at the University of Southampton in England because it has one of the first and largest IRs with a mandate policy employed at the institutional level, and we are interested in observing how a mandate policy has affected self-archiving. ${ }^{8}$ This is a group of scientists who can enjoy both types of repository, both of which are popular to the academic community.

\section{Background}

As early as 2001, Pinfield analyzed the application of self-archiving pre-prints in arXiv with the purpose of looking for implications for the design of infrastructure of a pilot institutional repository at the University of Nottingham. ${ }^{9}$ He was interested in learning how the experience of physicists could be used to run a multidisciplinary institutional service. The flexibility of the e-prints model and the adaptability of it to disciplines other than physics were explored. His work represented a common thought of digital professionals who have been concerned with IR development and management and would rely on a disciplinary culture of self-archiving to promote the participation of faculty in the adventure of the new scholarly publishing.

About five years later, self-archiving has not been able to bring a satisfactory number of content materials to the majority of IRs although a very few IRs are maintaining a sizable content that makes them look promising. ${ }^{10}$ Faculty still stay away from the self-archiving activities and do not show increasing interest in the efforts in the near future. ${ }^{11}$ Evidently, they have different attitudes toward sharing research results in subject-oriented online portals where people have similar research interests than in institutional online databases where people are connected by employment affiliation. ${ }^{12}$ Publishing research results electronically on the latter platform cannot bring much benefit to faculty in terms of professional promotion and scholarly exchange.

At the first, IR advocates argued that the main reason that faculty had not enthusiastically collaborated with their institutional repositories was because they were unaware of the existence of the repositories. ${ }^{13}$ The argument was then denied by subsequent studies that found the explanation was too simple to speak the truth. ${ }^{14}$ However, all believed that faculty's familiarity with open access self-archiving would help IRs accumulate content materials, ${ }^{15}$ although the belief lacked scientific evidence. "There is a direct correlation between willingness to self-archive and the existence of subject-based repositories... where there is a pre-existing culture of self-archiving e-prints in subject repositories, scholars are more likely to post research material on their own Web pages". ${ }^{16}$

A couple of studies have particularly focused on the exploration of disciplinary culture for the development of institutional repositories. These studies include an investigation of nine scientific disciplines across the United States and Canada, ${ }^{17}$ an international survey among senior journal authors, ${ }^{18}$ an author study on open access self-archiving, ${ }^{19}$ and interviews among selected faculty members at the University of California at Berkeley on their behavior, attitudes, and perceptions of the new scholarly 
communication systems. ${ }^{20} \mathrm{~A}$ more recent research about the non-use of Cornell University’s DSpace found that this well-known repository was largely under-populated and underused by Cornell faculty who showed little knowledge of and little motivation to use DSpace. ${ }^{21}$ This research also agreed with the theory of the disciplinary culture proposed before and stated that "cultural norms were regularly brought up as justification for behavior.” As a result, one would have observed that while it was essential that mathematicians took a normative and pragmatic approach to communicate scholarly, historians chose to publish monographs in a non-collaborative style and physicists considered peer-reviewed journal publications more as a sign of scholarly accomplishments than as an efficient channel of disseminating research findings and ideas.

At some institutions, the theory had provided the basis upon which approaches to plead faculty's contributions were developed. An example is the plan of several institutional repositories in Canada that would implement a pilot project to focus on a single discipline across institutions by collecting digital materials and developing a harvesting service to collect the materials. ${ }^{22}$ Upon successful implementation, practice of self-archiving by faculty in this discipline would serve as a sampler for other disciplines in the IR. Another example is Dutch's strategy of concentrating on working with some of the most prominent scholars at participating institutions to archive their research output in the IRs as a showcase to the rest faculty authors in the same institutions. ${ }^{23}$

A preliminary study on the assessment of self-archiving of different faculty members was recently taken to evaluate the theory of disciplinary cultures across seven institutional repositories in several countries. ${ }^{24}$ It, unfortunately, did not find solid evidence to support the theory. Instead, it found that there was not an obvious pattern of self-archiving experiences among four disciplines examined — chemistry, economics, physics, and sociology. Two of the disciplines, namely, economics and physics, have a long tradition of using subject repositories to share research results, while the other two, namely, chemistry and sociology, do not have a successfully operated repository for scholars. A tentative suggestion of the study disproved the importance of faculty's familiarity with self-archiving tradition for the success of IR content recruitment. Among the operational tactics of the IRs that have played a crucial role in the making of the content are a liaison system and a mandate policy. ${ }^{25}$

Apparently, all the studies of disciplinary culture relied on comparisons of self-archiving across academic disciplines. Evidence was obtained primarily by either asking questions to faculty authors or copying figures from repository records. The assumption of the inheriting behavior of faculty in self-archiving was never verified by comparing the difference of the actual practices by the same scholars between their contributions to subject repositories and to institutional repositories. The difference of self-archiving numbers across disciplines may be caused by many factors irrelevant to the attitudes and perceptions of faculty such as institutional policies and the implementation of metadata harvesting. On the other hand, tracing the practices of self-archiving of individual authors to see how scholars of the same discipline have worked with repositories in both their research community and institution will provide dissimilar set of information for a better understanding of disciplinary culture and help repository professionals make appropriate management decisions.

Additionally, those studies that emphasized content size of IRs did not examine the status of individual items. The figures they cited to suggest a disciplinary divide were merely the total numbers of deposits - 
too simple to accurately describe the complex situations in IR practice. For instance, the total number of IR deposits for an academic field will become more meaningful when it is compared to the number of faculty members in the department. Also, the number of articles published per faculty member is an important comparative factor. Publishing is easier in some fields than others because of the differences in knowledge and disciplinary structures.

\section{Hypothesis}

The disciplinary culture theory emphasizes how the tradition of information acquisitions and dissemination of a discipline has shaped the behavior of its scholars for joining the adventure of the new publishing system. Its manifestation is the consistency of scholars in the practices of self-archiving in both SRs and IRs. The theory also underlines that scholars' involvement in the accumulation of a repository's content will make it possible for them to get involved in the activities of another repository. The development of SRs was about ten years earlier than IRs, and in existence for only few disciplines. Therefore, in a field where a SR was in practice before an IR, a scholar who has been active contributing to the SR will possibly bring the same familiarity and enthusiasm to the IR. One will expect to observe the same (or similar) amount of deposits in both the SR and the IR by this scholar.

\section{Methodology}

\subsection{Research method}

For research data of the institutional repository, the University of Southampton was selected as the subject and its repository e-Prints Soton was checked for physics articles. From the interface of e-Prints Soton, ${ }^{26}$ the "browse" function was referred to look at articles sorted by "school and research group" under which the School of Physics and Astronomy was examined and a total of about 500 items were found as of February 2008. Each item was manually checked and its metadata was recorded including author names, article title, year of deposition, name of depositor, full-text availability, article format, and external link(s). Because all articles under this school were grouped into four categories (general, astronomy and space science, quantum, light and matter, and high energy physics) that created some across-category displays of articles, the total recorded items were more than 500, including articles, book chapters, conference proceedings, theses, working papers, etc.

From the website of the school a list of its current physicists, including both teaching and research faculty, was then checked and recorded. A total of 51 names were found regardless of the status and rank of each individual in the school.

For research data of the subject repository, arXiv was checked by searching personal names that were in this Southampton's physicist list. Each of the names was entered for an author search with the interest of knowing how many articles of a particular scholar at Southampton have been archived in arXiv and who did the deposition. arXiv provides a "submission history" metadata to reflect the depositorship of an article. A tricky part of these searches was name variations. The naming convention of arXiv is "first initial, second initial, and full last name without a dot following the initials”. ${ }^{27}$ However, authors' names have been entered differently which may have been the result of separate depositors, different dates of 
deposition, or various appearances of an author's name in the articles. A great deal of time was spent searching articles by using variations of one's name, e.g., initial versus full name; and extra work was done to verify the current affiliation of an author as well as his/her working history in order to avoid the problem of name confusion, particularly for the common names. Upon a careful verification, a total of 1,556 articles authored or co-authored by Southampton physics faculty was recorded from arXiv.

\subsection{Limitations}

Even though great care was made to verify name variations of the authors, the accuracy level of the searching is still imperfect. Tracing professional history of a scholar might not work very well because the SR article search was based on a list of current physicists in the school whose former employment may be unknown. The webpage of the school did not provide a CV for most scholars, making the verification of one's previous employment difficult. To raise the accuracy level of the name search, an attempt was made to not only analyze the variations of a name, but also to compare his/her research subjects and co-authorship should confusion occur.

Especial attention was paid to the examination of some articles in Southampton's e-Prints Soton to determine the author's relationship to the institution, namely, articles by one whose name is not associated with the school but this article is listed under the category of physics and astronomy. This author might have previously worked there or been a student of the school. However, such uncertainties are very rare and can be considered acceptable because the aim of this study is to figure out how enthusiastic the physicists have been about contributing articles to their IR depending on their familiarity level to self-archiving through a long-run SR repository, but not the reverse.

\section{Findings and discussion}

\subsection{The subject culture theory}

Based on a list of 51 names currently affiliated with the School of Physics and Astronomy, searching was done in a SR and an IR to retrieve articles authored as well as deposited by them with an attention being paid to articles deposited by them. Data shows that as many as 453 articles in arXiv have been identified as self-submissions by current Southampton physicists; and 24 Southampton's names are found in the submission list. Less than half of the total physicists have a history of making contributions to the SR. In comparison to the SR, self-archiving is practiced by only 21 scholars in their own IR with a total of 240 articles (Fig. 1). The number of articles self-archived in the IR counts for only about half of articles selfarchived in the SR. This may indicate that the faculty authors are not as interested in working with their IR as with a SR. Fig. 2 shows a comparison of the numbers of self-archiving participants between arXiv

and e-Prints Soton. Please note that the numbers of the article authors in both the SR and the IR are higher than the numbers of self-archiving participants. This is normal because an author may self-archive more than one article, or an article may be archived by a co-author who is not Southampton related. Please also note that more faculty names are found in the IR than in the SR. Metadata reveals that many faculty have never participated in self-archiving in the IR and whose articles are deposited by a third party - a mediated archiving. 


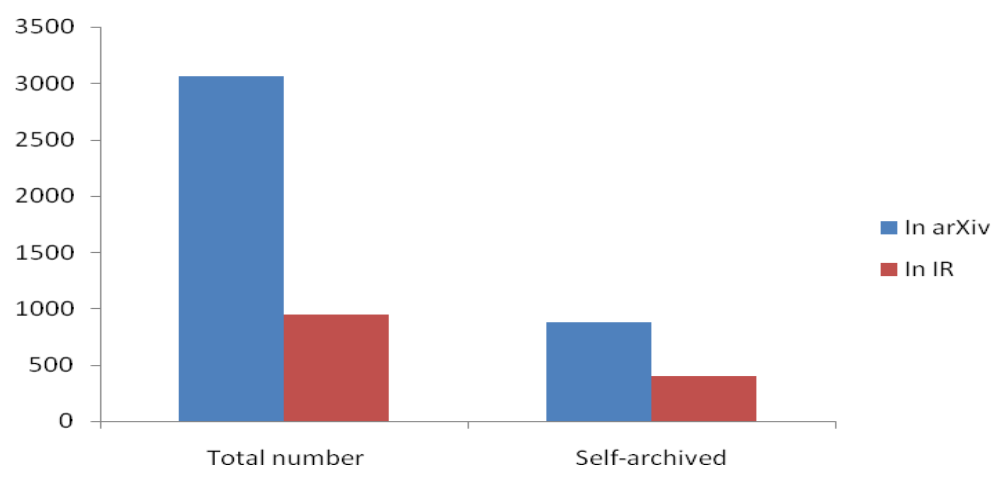

Figure 1. Comparison of the total numbers of articles archived versus self-archived between arXiv (SR) and e- Prints Soton (IR). Please note that the total number of articles archived in IR is more than the actual total number 500. This is because the former is calculated by checking authors' names. One article may be co-authored by more than one Southampton physicist. This is for the purpose of comparison and is acceptable given the fact that the total number of articles archived in arXiv is counted the same way.

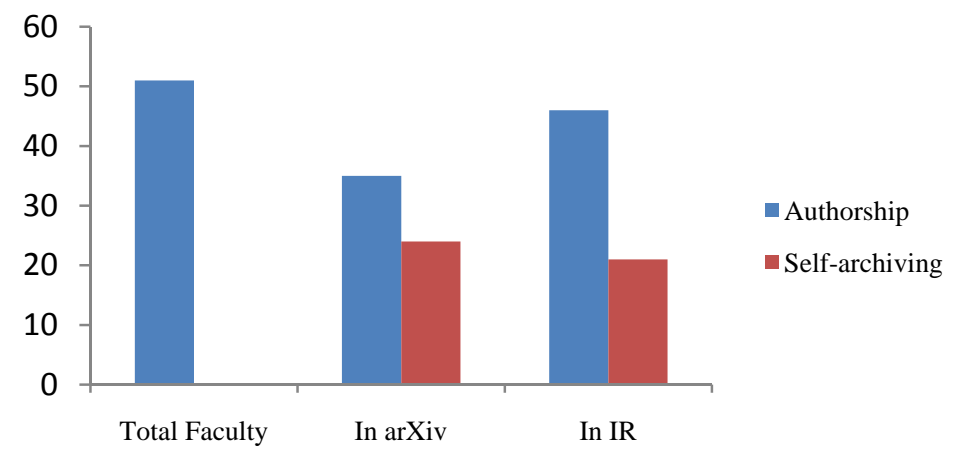

Figure 2. Comparison of numbers of faculty authors whose names are found in arXiv (SR) and e-Prints Soton (IR)

Because the hypothesis holds that if one has experience self-archiving articles in a SR, s/he will be more likely to contribute to an IR than one who lacks the experience, it is important to see from the data collected if the SR contributors are also the IR contributors. The finding is interesting — there are 12 faculty authors who have done self-archiving in arXiv but have not made any contribution to the IR of their own institution. This number counts for half of the arXiv contributors. Particularly, the most enthusiastic SR depositors in the faculty list, namely those who have archived more than twenty articles in arXiv, tend to generate much less, if any, of their IR content. Also interesting is that nearly half of the IR contributors have never worked with arXiv for self-archiving previously. Table 1 provides some numbers for the purpose of comparison. If these numbers are really meaningful, the suggestion will be that there lacks concrete evidence to support a correlation between the two practices, and hence the hypothesis is rejected.

But this by no means indicates that most scholars are not familiar with the practice of self-archiving in arXiv. Rather, arXiv has been well referenced when external link of a deposit in the IR is provided. Approximately seven percent of all articles in the IR has an external link to the full text in arXiv; and another seven percent links to PubMed for a full text. This finding, alongside the figures mentioned above, 
may suggest that scholars have been aware of the existence of both subject-based and institutional repositories but just do not want to waste time on repeating self-archiving activities if their articles are already available in one repository database. Otherwise, we will not observe the obvious ignorance of one repository by some authors who are energetic in the other repository. Except for a couple of authors, those who have deposited a lot in arXiv seldom contribute to e-Prints Soton, and vice versa. If a comparison of the total deposits between arXiv and e-Prints Soton is made, most faculty authors seem to prefer to work with the SR than with the IR (Fig. 1). It remains unknown if this is related to their attitude toward selfarchiving in one particular type of repository, or it is just because of the length of the history of different repositories.

Before e-Prints Soton was created in 2003 most Southampton physicists had already been familiar with and made contributions to arXiv. The data here confirms an assumption mentioned above that scholars seem to avoid wasting time on repeating self-archiving if their articles have been available in one repository, because although many of these early arXiv contributors also participated in self-archiving in the IR after it became available, they have been selective in working with either of the repositories, but not both, as their primary deposition location.

\begin{tabular}{|c|c|c|}
\hline & arXiv Contributors & IR Contributors \\
\hline arXiv Contributors & 24 & 9 \\
\hline IR Contributors & 12 & 21 \\
\hline
\end{tabular}

Table 1. Comparison of arXiv contributors and IR contributors

Beside the external links, some other data are also worth mentioning. Among others, the availability of full text may be able to tell the usability of a repository. As a normal practice, arXiv requires the deposition of full text and seldom has abstract-only items in existence. On the other hand, e-Prints Soton has a percentage of abstract-only items as high as sixty two percent of the total articles (310 items, see Fig. 3). Although some of the abstract-only items have a link to the full text in an external online source, such a link may have become a dead end because of the retirement of an external website and, most possibly, because of the restricted access to a subscription-based database. Of the 468 items with an external link to the full text, more than half (253 items, see Fig. 3) have a dead link. This large percentage of unavailable full texts can easily wear out readers' interest in using the repository, which can be an interesting topic to explore in the future.

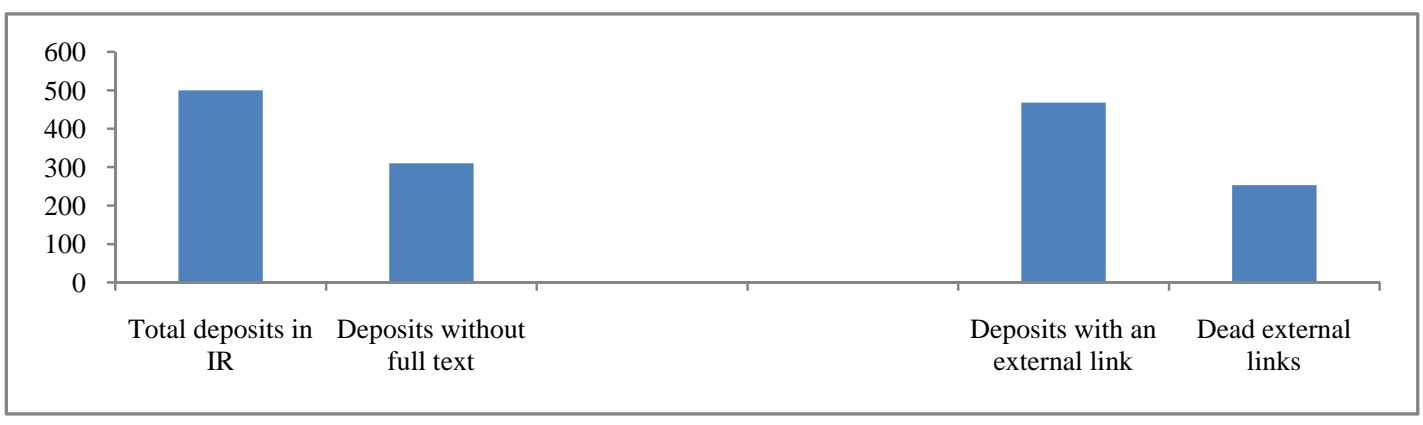

Figure 3. Status of full text availability in e-Prints (IR) and percentage of dead external links 
By knowing the small percentage of full-text deposits, we may want to pay attention to the importance of quality control in the content of institutional repositories. This is particularly essential to the increasing implementation of mandate policies. One possible challenge with such an implementation may be that some authors are indifferent towards self-archiving or have unresolved concerns on issues like copyrights, but have to do so under the pressure of institutional requirements. Their depositing work may not follow the exact archiving procedure and result in less-useful deposits with very few metadata entries and without a full text loaded.

Back to the data of depositors in e-Prints Soton, out of a total of 500 articles more than 310 are actually deposited by non-authors, consisting of sixty-two percent. These depositors are identified as either "import" or with a name by the title of "Ms." or "Mrs." who are not authors of any article archived. Given the fact that there are only seven of such non-author depositors and each is related to many deposits (Table 2), one may be able to believe that they have been assigned to do the archiving on behalf of faculty authors or they have been utilizing certain mechanism to automatically import articles from somewhere else. Mediated depositing has been a salvation to the unsatisfactory performance of self-archiving in many institutional repositories. Yet, it is unrealistic to expect a third-party depositor to read all articles before uploading them and filling out metadata information. Then, how can we expect the subject terms of any article being entered to precisely represent the topics of the article so that it will be suitably found in article retrievals? (Not every journal asks their articles to have necessary keywords.) Therefore, although such mediated archiving can be loosely defined as "self-archiving", ${ }^{28}$ the quality of their depositing may be different. Also, a subject culture theory is in no way applicable to the example here.

\begin{tabular}{|l|c|c|c|c|c|c|c|}
\hline Depositor & $\begin{array}{c}\text { Group, } \\
\text { Astronomy }\end{array}$ & $\begin{array}{c}\text { Lawrence, } \\
\text { Mrs. Nicky }\end{array}$ & $\begin{array}{c}\text { ORC, } \\
\text { Import }\end{array}$ & $\begin{array}{c}\text { Research Centre, } \\
\text { Optoelectronics }\end{array}$ & $\begin{array}{c}\text { Searle, Ms } \\
\text { Christine }\end{array}$ & $\begin{array}{c}\text { SES, } \\
\text { Import }\end{array}$ & $\begin{array}{c}\text { SOC, } \\
\text { Import }\end{array}$ \\
\hline $\begin{array}{l}\text { Deposit } \\
\text { Number }\end{array}$ & 45 & 10 & 7 & 22 & 89 & 124 & 13 \\
\hline
\end{tabular}

Table 2. Major non-author depositors in the IR with a total of 310 deposits

\subsection{Web 2.0 and user-generated content}

Since the first O’Reilly Media Web 2.0 conference held in 2004, Web 2.0 has become a new generation of web-based networking that facilitates interactions between clients and clients and between clients and servers, and encourages the participation of end-users in information sharing. ${ }^{29}$ Web 2.0 is now providing a different concept of internet platform through which people can communicate with each other and contribute to existing datasets. "Web 2.0 is a knowledge-oriented environment where human interactions generate contents that are published, managed and used through network applications in a serviceoriented architecture." ${ }^{30}$ Surely true is that digital repositories satisfy the definition of Web 2.0 with regard to their mechanism of accumulating content materials. The activity of self-archiving highlights the participation of users in information exchange through programmed web applications. A digital repository, either subject-based or institutional, is a special type of digital library. Both share the same goals and utilize similar technologies in data preservations and disseminations, but differ in the way that data 
acquisitions are undertaken. The user-as-contributor nature of digital repositories supports interactivity and provides reference for the improvement of the exhibition-oriented digital libraries in accommodating more dynamic services. Hence, the understanding of user behaviors toward creating content for digital repositories and particularly, the influence of technologies on the behaviors, will benefit digital libraries for future development.

Simplicity is an important consideration when a repository application is designed. The ease of use of an application can encourage the participation of faculty users in the creation of repository content. A typical application requires users to upload a digital file to the server and at the same time fill out necessary metadata elements which range from article title, author name, and journal name to subject terms, bibliography, abstract, and the like in order to complete one deposition. It is believed that this complicated deposition process has been too much a task for faculty authors who are usually busy in research and teaching. ${ }^{31}$ According to these studies this complicity is one of the reasons why the majority of institutional repositories have failed in collecting a large amount of items so far.

Some repository advocates disagree with this assumption and insist that current repository applications are simple to use and won't consume too much of a depositor's time. One study analyzing repository log files found that the time required for the deposition of a paper is only about $10 \mathrm{~min}$, and even shorter for an experienced depositor. ${ }^{32}$ Another study also denied possible technical difficulties in performing selfarchiving activity. ${ }^{33}$ Yet, our study reveals that faculty authors are indeed not enthusiastic about archiving their articles into their institutional repository even though they are familiar with self-archiving practice and know how to quickly archive an article. Technology seems not to be a major concern in the process of content generation by users, although poor design of a repository's interface will definitely discourage users from taking part in the interaction.

Self interests of scholars motivate interactions while requirement from policies is another factor affecting scholars' participation. The latter may pressure faculty authors on agreeing to contribute their research results to a repository; but the contribution may be done by a third party such as an assistant or secretary on behalf of the authors as demonstrated in this study. Broadly speaking, this may also be considered as user participation, depending on how "user” is defined, and may also be described as self-archiving, depending on how "self" is defined. The question is, however, if such detour interactions can continue without the real involvement of authors who are also readers.

Successful stories of interaction in blogging underline the importance of self interests in stimulating online communication. Mandate policies are not even common practice for IRs and have many unsolved issues such as copyrights. They are totally unknown to the digital library community. Therefore, by having learned from the experience in the operations of IRs, designers and managers of digital libraries should concentrate on users' needs and interests if interactivity is aspired.

\section{Conclusion}

Previous studies have found that physicists have done no better than scientists in other subjects in making contributions to their institutional repository. ${ }^{34}$ Within the subject of physics, data collected from selfarchiving in a SR and an IR seem to not support the subject culture theory that scholars' familiarity with 
self-archiving through practices in a SR will ensure their enthusiasm about self-archiving in the repository of their own institution. Instead, evidence reveals that when an article has been presented in one repository, the author(s) will be hesitant to make it repeatedly available in a second repository. Also, scholars who have been energetic in submission to a SR may not necessarily be activists in an IR, and vice versa.

Personal interest in an IR means much more for the development of institutional repositories than being obligated by a mandate requirement. Otherwise, people may just make deposit numbers by simplifying the depositing process and providing inadequate data. Any repository with unqualified deposits such as a large number of non-full-texts will keep users away from using it. Online communication is necessitated by contributor-to-reader interactions. Therefore, when people are happy with the success of mandate policies in digital repositories, it is equally important to carry out quality control over repository content by setting up guidelines for self-archiving. IR managers will need to improve their technology and services and make their repositories attractive in both content and presentation, rather than relying solely on policies.

Physicists at Southampton are the subjects of this research. The sample size is relatively small. More intensive studies of this type will be able to shed better light on self-archiving practice in both subjectbased and institutional repositories and provide more evidence for repository managers to enhance their services. Both quantitative analyses and qualitative studies such as questionnaires and interviews will help understand how scholars perform self-archiving in and what expectations readers have for a repository.

\section{Notes and References}

1. Peter Suber, Open access news: news from the Open Access Movement. Available http://www.earlham.edu/ peters/fos/2007/12/oa-mandate-at-nih-now-law.html (accessed May 7, 2008).

2. Patricia Cohen, “At Harvard, a Proposal to Publish Free on Web,” The New York Times, February 12, 2008. Available http://www.nytimes.com/2008/02/12/books/12publ.html?_r=1\&oref=slogin (accessed May 7, 2008); News blog, "Harvard faculty adopts open-access requirement," The Chronicle of Higher Education, February 12, 2008. Available http://chronicle.com/news/article/3943/harvard-faculty-adopts-open-access-requirement (accessed May 7,2008); Peter Suber, Open Access News (2008). Available http://www.earlham.edu/ peters/fos/fosblog.html (accessed May 7, 2008).

3. Arthur Sale, “The Acquisition of Open Access Research Articles," First Monday 11, no. 10 (October 2006). Available http://www.firstmonday.org/issues/issue11_10/sale/index.html (accessed May 7, 2008).

4. Queens Margaret University, Open Access self-archiving policy. Available http://www.eprints.org/openaccess/policysignup/fullinfo.php?inst=Queen\%20Margaret\%20University\%2C\%20 Edinburgh (accessed May 7, 2008).

5. The Irish Research Council for Science, Engineering \& Technology, Open Access Policy. Available http://www.ircset.ie/news/releases/080501_OpenAccessPolicy.html (accessed May 7, 2008).

6. EPrints.org, ROARMAP (Registry of Open Access Repository Material Archiving Policies). Available http://www.eprints.org/openaccess/policysignup/ (accessed May 7, 2008). 
7. Henk F. Moed, “The Effect of 'Open Access' on Citation Impact: An Analysis of ArXiv's Condensed Matter Section,” Journal of the American Society for Information Science and Technology 58, no. 13 (August 2007), pp. 2047-54.

8. JISC, Guide For HEI Managers — Ensuring a successful institutional repository. Available http://www.lboro.ac.uk/departments/ls/disresearch/poc/pages/HEIguide-IRlaunch.html (accessed May 7, 2008).

9. Stephen Pinfield, "How do Physicists Use an E-Print Archive? Implications for Institutional E-Print Services," D-Lib Magazine 7, no. 12 (December 2001). Available http://www.dlib.org/dlib/december01/pinfield/12pinfield.html (accessed May 7, 2008).

10. Jingfeng Xia and Li Sun, "Assessment of Self-Archiving in Institutional Repositories: Depositorship and FullText Availability,” Serials Review 33, no. 1 (March 2007), pp 14-21.

11. Alma Swan and Sheridan Brown, Open Access Self-archiving: An Author Study. Report, Key Perspectives Limited. May 2005. Available http://eprints.ecs.soton.ac.uk/10999 (accessed May 7, 2008).

12. C. Judson King, Diane Harley, Sarah Earl-Novell, Jennifer Arter, Shannon Lawrence, and Irene Perciali, Scholarly Communication: Academic Values and Sustainable Models. Report, Center for Studies in Higher Education, University of California, Berkeley, July 2006. Available http://cshe.berkeley.edu/publications/docs/scholarlycomm_report.pdf (accessed May 7, 2008).

13. Morag Mackie, "Filling Institutional Repositories: Practical Strategies from the DAEDALUS Project,” Ariadne 39 (April 2006). Available http://www.ariadne.ac.uk/issue39/mackie (accessed May 7, 2008).

14. Alma Swan and Sheridan Brown, “Authors and Open Access Publishing," Learned Publishing 17, no. 3 (July 2004), pp. 219-24.

15. Mary Ann Kennan and Concepción S. Wilson, "Institutional Repositories: Review and an Information Systems Perspective,” Library Management 27, no. 4/5 (June 2005), pp. 236-48; Clifford A. Lynch, "Institutional Repositories: Essential Infrastructure for Scholarship in the Digital Age," Portal: Libraries and the Academy 3, no. 2 (April 2003), pp. 327-36; Stephen Pinfield, “Self-Archiving Publications," In International Yearbook of Library and Information Management 2004-2005: Scholarly Publishing in an Electronic Era, edited by Gary Gorman and Fytton Rowland (London: Facet Publishing, 2004): 118-45; Mark Ware, Pathfinder Research on Web-based Repositories —Final Report. (Bristol, UK: Publisher and Library Learning Solutions, 2004).

16. Theo Andrew, “Trends in Self-Posting of Research Material Online by Academic Staff,” Ariadne 37 (October 2003). Available http://www.ariadne.ac.uk/issue37/andrew (accessed May 7, 2008).

17. Ibironke Lawal, "Scholarly Communication: the Use and Non-use of E-Print archives for the Dissemination of Scientific Information,” Issues in Science and Technology Librarianship, Fall 2002. Available http://www.istl.org/02-fall/article3.html (accessed May 7, 2008).

18. Ian Rowlands and Dave Nicholas, New journal publishing models: an international survey of senior researchers. A CIBER Report for the Publishers Association and the International Association of STM Publishers, September 2005. Available http://www.ucl.ac.uk/ciber/ciber_2005_survey_final.pdf (accessed May 7, 2008).

19. Swan and Brown, Open Access Self-archiving: An Author Study.

20. King, et al, Scholarly Communication: Academic Values and Sustainable Models. 
21. Philip M. Davis and Matthew J.L. Connolly, "Institutional Repositories: Evaluating the Reasons for Non-use of Cornell University’s Installation of DSpace,” D-Lib Magazine 13, no. 3/4 (March/April 2007). Available http://www.dlib.org/dlib/march07/davis/03davis.html (accessed May 7, 2008).

22. Kathleen Shearer, "The CARL Institutional Repositories Project: a Collaborative Approach to Addressing the Challenges of IRs in Canada,” Library Hi Tech 24, no. 2 (2006), pp. 165-72.

23. Martin Feijen and Annemiek van der Kuil, "A Recipe for Cream of Science: Special Content Recruitment for Dutch Institutional Repositories,” Ariadne 45 (October 2005). Available http://www.ariadne.ac.uk/issue45/vanderkuil/ (accessed May 7, 2008).

24. Jingfeng Xia, “Assessment of Self-Archiving in Institutional Repositories: Across-Disciplines,” Journal of Academic Librarianship 33, no. 6 (December 2007), pp. 647-54.

25. Arthur Sale, “Comparison of Content Policies for Institutional Repositories in Australia," First Monday 11 (April 2006). Available http://www.firstmonday.org/issues/issue11_4/sale/index.html (accessed May 7, 2008).

26. Southampton’s Soton Eprints. Available http://eprints.soton.ac.uk (accessed May 7, 2008).

27. ArXiv. Available http://arxiv.org/multi?group=grp_physics\&\%2Ffind=Search (accessed May 7, 2008).

28. Self-Archiving FAQ. Available http://eprints.ecs.soton.ac.uk/10635/1/index.html (accessed May 7, 2008).

29. Tim O'Reilly, What is Web 2.0. Available http://www.oreillynet.com/pub/a/oreilly/tim/news/2005/09/30/whatis-web-20.html (accessed May 7, 2008).

30. Web 2.0 Preface. Available http://lindipendente.splinder.com/post/15354690/World+2.0 (accessed May 7, 2008).

31. Mackie, "Filling Institutional Repositories: Practical Strategies from the DAEDALUS Project.”

32. Leslie Carr and Stevan Harnad, "Keystroke Economy: A Study of the Time and Effort Involved in Selfarchiving,” (March 2005). Available http://eprints.ecs.soton.ac.uk/10688/ (accessed May 7, 2008).

33. Swan and Brown, Open access self-archiving: an author study.

34. Xia, "Assessment of Self-Archiving in Institutional Repositories: Across-Disciplines.” 